\title{
AUTOTUNE OF PID TEMPERATURE CONTROL BASED ON CLOSED-LOOP STEP RESPONSE TESTS
}

\author{
D.C. Sheats ${ }^{1}$, Z.S. Roth ${ }^{2}$ J.W. Snyder ${ }^{1}$ \\ ${ }^{1}$ Scientific Instruments, Inc. \\ West Palm Beach, Florida, 33407, USA \\ ${ }^{2}$ Florida Atlantic University \\ Boca Raton, Florida, 33431, USA
}

\begin{abstract}
A novel PID control autotune technique for temperature control systems operating in the cryogenic regime is described. The PID parameter tuning is based on a sequence of closed-loop step response tests utilizing the measured peak overshoot values and the time between the first peak and first dip of the step response. The performance of this technique is compared to that of a Ziegler-Nichols test. This empirically developed technique has proven to work well for certain types of cryogenic applications and we explore its potential for broader use.
\end{abstract}

KEYWORDS: PID, autotune, temperature control

PACS: 07.20.Mc, 07.05.Dz, 89.20.Bb

\section{INTRODUCTION}

Variations of the Proportional, Integral, and Derivative (PID) control algorithm are used in many different industries to control a wide variety of processes. The popularity of PID stems from its simplicity and flexibility. All one needs to implement PID control is to define a set point variable (SP), a process variable (PV), and a meaningful way to represent the error in terms of these two and the algorithm does the rest. While this control method is straight forward to implement, the tuning of the algorithm can be very complex. In fact, the tuning of a PID controller to a specific process often times seems like "black magic" to the uninitiated. It is to these users that a PID auto-tuning technique is of the greatest value. We have developed one such technique for use with a cryogenic temperature controller and describe its application here. 
A typical cryogenic apparatus that has been designed for active temperature control consists of a block, a heater, and a thermometer. The block is cooled at all times either passively, through contact with a cryogenic liquid, or actively through a refrigeration process. The objective of control is to bring the block's temperature to a specific value and maintain it there. This is done by applying an appropriate amount of power to the heater while measuring the temperature with a thermometer. It therefore makes sense to define the process variable as the output of the thermometer and set-point as the desired value of the block temperature.

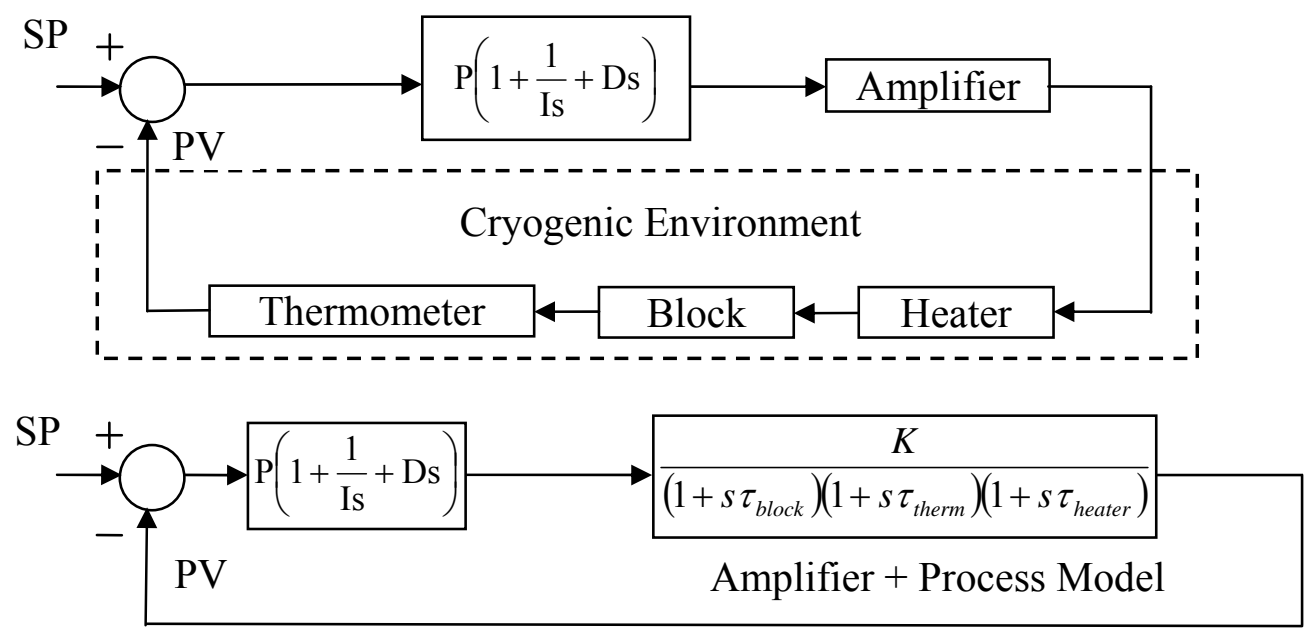

FIGURE 1. Control loop diagrams of the M9700 temperature controller with a generalized cryogenic load.

The process that relates the block temperature to the heater power is typically modeled as a third-order linear system. Such a model must take into account the heat capacities of the heater, block and sensor, and the thermal resistances of the blockcryogenic bath path, heater-block connection, and the thermometer-block connection. These capacities and resistances translate into three time constants in an all-pole heater power to thermometer temperature transfer function [1]. Typically, the process gain, $\mathrm{K}$, is equal to the thermal resistance between the block and cryogenic bath and the three time constants are represented as $\tau_{\text {block }} \approx \mathrm{R}_{\text {block-bath }} \mathrm{C}_{\text {block}}, \tau_{\text {heater }} \approx \mathrm{R}_{\text {heater-block}} \mathrm{C}_{\text {heater }}$, and $\tau_{\text {thermometer }}$ $\approx \mathrm{R}_{\text {block-thermometer }} \mathrm{C}_{\text {thermometer. }}$. The block's time constant is typically much larger than either of the other time constants and may be in the order of magnitude of tens or hundreds of seconds, whereas the faster constants may be in the order of a few milliseconds. The open-loop step response of such processes has the familiar S-shape featuring a gain $\mathrm{K}$, an approximated pure time delay $\mathrm{L}$ and a time constant $\tau$ that typically represents the dominant time constant, that of the block. The value of $\mathrm{L}$ represents the other, shorter time constants in the system. Measurement of K, L and $\tau$ constitute the open-loop ZieglerNichols (Z-N) Step-Response Test. The Z-N recommended PID control parameters are functions of $K, L$, and $\tau[2,3]$. Such tuning is valid only for processes that indeed feature an S-shaped step response. Auto-tuning according to a Ziegler-Nichols test in open-loop may involve the automated measurement of $\mathrm{K}, \mathrm{L}$, and $\tau$, followed by an automatic computation of the P, I and D control coefficients. In closed-loop, Z-N tuning requires an instability borderline search where the $\mathrm{P}$ value is increased until sustained oscillations are induced. The P value is reduced by an appropriate amount and the I and D terms are calculated from the period of the oscillations.

Control researchers, over the years, have tried to optimize the selection of PI and PID control parameters, given the values of $\mathrm{K}, \mathrm{L}$, and $\tau$, according to various quantitative 
performance criteria. The most complete compilation of formulas for the PID gains may be found in [4].

The accuracy of Ziegler-Nichols open-loop tuning depends on errors in measuring the time delay and time constant parameters. The delay parameter L, in particular, may be difficult to measure in systems where $\mathrm{L}<<$. Indeed, in the cryogenic environment described above, the block's time constant is often one or more orders of magnitude larger than the time constants of the heater and the thermometer. The P-control closed-loop Ziegler-Nichols test can be quite time consuming due to the need to establish a sustained oscillation in the loop.

The question of when one can use a PI controller, and when a PID controller must be used, does not have an explicit answer in most literature sources. In [5] it is argued that the controller, for processes which exhibit a single dominant time constant, should not be more complicated than a PI. According to [5], a PID controller is needed whenever the system has two dominant time constants. According to that, a typical cryogenic temperature control system may be controlled by a PI controller. However, the analysis is based on an implicit assumption that the process is always linear. Amplifier saturation, uni-directional control action nonlinearity and parameter variation effects, common to cryogenic applications, are shown to necessitate the use of a PID controller, over a PI controller, as a better practical controller.

The time-domain control law used is given by equation 1 where $u$ represents the heater output, $t$ is time, and $e(t)$ the difference PV-SP.

$$
u=P e(t)+\frac{P}{I} \int_{o}^{t} e(t) d t+P D \frac{d e(t)}{d t}
$$

An implementation of this for digital control involves sampling the temperature, $\Theta$, every $T_{s}$ seconds (e.g. $\Theta(0), \Theta\left(T_{s}\right), \Theta\left(2 T_{s}\right)$, etc...). For the controller in test the value of $T_{s}$ was 0.133 seconds. Error values were accumulated such that at $t=0, e(k)=e(0)$; at $t=$ $T_{s,} e(k)=e(1)$ and so on. With this, the control law takes the form of equation 2.

$$
u(k) \approx P e(k)+\frac{P T_{s}}{I} \sum_{k} e(k)+P D \frac{e(k)-e(k-1)}{T_{s}}
$$

\section{AUTOTUNING PROCEDURE}

The iterative step-by-step PID tuning procedure, summarized below, was obtained through exhaustive experimentation at Scientific Instruments, Inc. [6]. We shall hereafter refer to the procedure as the SI Method. It is an empirical result. The authors have been working on a theoretical explanation to this particular method, and related class of methods.

The step-by-step auto-tuning procedure is as follows:

Step 0 . The system starts at a default set of PID parameters. In the case of multiple tuning procedures, the system's initial settings will be the same as the last used set of parameters.

Step 1. The system temperature must be stable to begin the tuning cycle. This typically requires a heater output and a non-zero I term. The best performance is realized by stabilizing the system at a temperature close to that where control actions are to occur. 
Step 2. The system set-point is increased by $2 \mathrm{~K}$ and the heater output is driven with "P only" control. The I term is held constant at the value used to achieve stability in Step 1 and the D term is set to zero. No integration is done at any point in the tuning experiment.

Step 3. The value of the maximum attained at the first peak (overshoot) is measured and the system is allowed to stabilize at the original set-point.

Step 4. If the overshoot is below $1.2 \mathrm{~K}$, the $\mathrm{P}$ term is adjusted up by $50 \%$. If the overshoot is above $1.2 \mathrm{~K}$, the $\mathrm{P}$ term is adjusted down by $50 \%$. The set-point experiment of step 3 is repeated with the new $\mathrm{P}$ value.

Step 5. The peak overshoot at the second trial is measured and a linear interpolation between the two trials is done to determine the value of $\mathrm{P}$ that would result in an overshoot of $1.2 \mathrm{~K}$. This value becomes the new $\mathrm{P}$ value and is used in successive tests.

Step 6. Using the new $\mathrm{P}$ value from step 5, another step test is performed and the time, $\Delta \mathrm{t}$, between the first peak and first dip is recorded.

Step 7. The controller's I term is set to the oscillation period of step $6, \mathrm{I}=2 \cdot \Delta \mathrm{t}$.

Step 8. The controller's D term is set to one quarter the I term, $D=I / 4$.

Comments: (a) The empirical search that led to the above tuning procedure revealed the special role played, during the tuning test, by a step response that has peak overshoot of around $60 \%$. As the proposed tuning test does not advocate reaching a step response steady-state, it is clear that a search for an overshoot of $1.2 \mathrm{~K}$ (equivalent to $60 \%$ of $2 \mathrm{~K}$ ) is easily implemented. Furthermore, the linear interpolation process done between the first two overshoot values predicts a $1.2 \mathrm{~K}$ overshoot, but in reality the overshoot obtained in trial 3 is only in the vicinity of this desired value. The auto-tuning performance seems fairly robust to all these procedural inaccuracies. (b) The above tuning procedure, done with a set-point of $2 \mathrm{~K}$, works successfully for set-points that are as large as $10 \mathrm{~K}$. There are applications in which even larger set-point changes are sometimes issued, but in these cases the heater quickly reaches it upper saturation limit, and stays there for a while, until the temperature approaches the desired level. This type of "largesignal controller wind-up" is not addressed here.

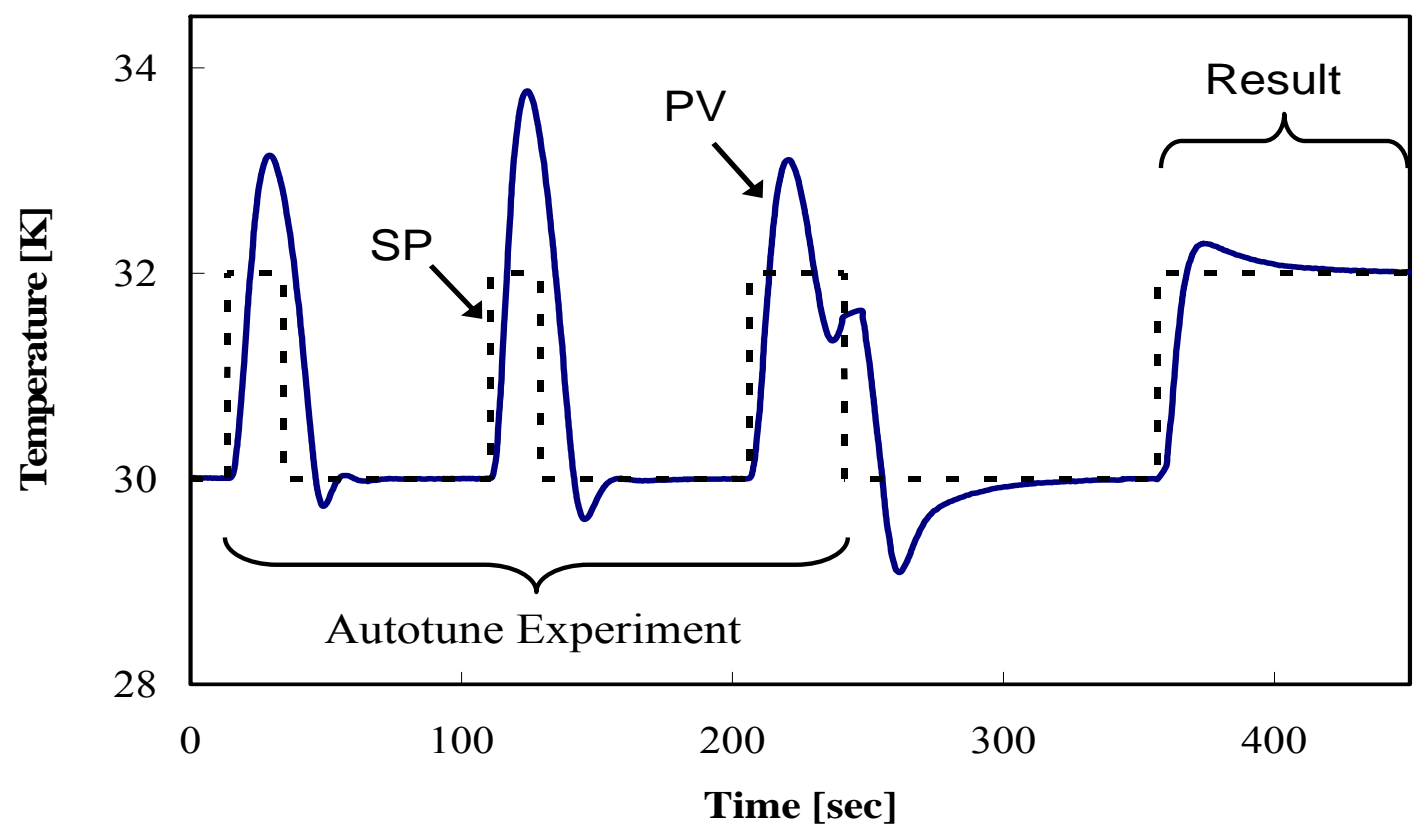

FIGURE 2. A typical auto-tuning experiment where the peak overshoot and time between first peak and first dip in the temperature (PV) are measured for a $2 \mathrm{~K}$ change in set point (SP). The resulting response, with updated PID values, is shown to the right of the figure. 
A typical tuning experiment, as conducted on the SI M9700 Temperature Controller, is shown in FIGURE 2 and serves to illustrate the tuning procedure. At T=30K the first overshoot occurs at $\mathrm{t}=29.1 \mathrm{~s}$ has a value of $1.149 \mathrm{~K}$. Shortly after, the system is returned to $\mathrm{T}=30 \mathrm{~K}$. In trial 2 , the overshoot (at $\mathrm{t}=124.5 \mathrm{~s}$ ) is $1.774 \mathrm{~K}$. Again, the system is reset. The actual peak overshoot, obtained in trial 3 is only $1.103 \mathrm{~K}$ (at $\mathrm{t}=221.1 \mathrm{~s}$ ). The first dip is $0.657 \mathrm{~K}$ below the set-point command of $\mathrm{T}=32 \mathrm{~K}$ and occurs at $\mathrm{t}=237.0 \mathrm{~s}$. We measure $\Delta \mathrm{t}=15.9 \mathrm{~s}$.

\section{ASSESSMENT OF CONTROL PERFORMANCE}

Many control tuning paradigms address "optimality" in some way. The SI Method aims at "acceptability" rather than optimality. There is no attempt to create the fastest possible closed-loop response, a no overshoot, or even a minimum overshoot response. The development goal was to create an algorithm that determined acceptable values for P, $\mathrm{I}$, and D quickly and reliably. The criteria that we use to evaluate the algorithm must include its reliability, process time, ease of implementation. Each of these can be considered alone as well as in comparison with other proven methods.

To assert reliability, one must perform exhaustive experiments as well as computational simulations. The SI Method has been implemented successfully on over one hundred systems worldwide. In one isolated case the tuning method provided suboptimal performance when a user connected two de power supplies in series in order to provide greater power to the heater. The second power supply introduced an extra time constant and this particular case is addressed in the simulation section below.

The first set of experiments compared PID control values, obtained by the new tuning method, to ones obtained via classic step-response and closed-loop oscillation Ziegler-Nichols tests. FIGURE 3 shows $\mathrm{P}$ and I values obtained by the three tuning methods, over the temperature range of $15-90 \mathrm{~K}$.

We see that the SI Method recommends the same P control values as the Z-N method, at the low temperature range $(15-40 \mathrm{~K})$, and a significantly lower $\mathrm{P}$ value at the higher temperature range. On the other hand, the SI method tends to use relatively large I (and consequently D) control values, compared to the Z-N method.

The process time for tuning depends heavily on the system and its characteristics. Large thermal masses and low cooling powers will cause the tuning process to take longer times. As can be seen in FIGURE 2, the process is quite quick when performed at a temperature where the cryostat can be considered to be in its normal operating range. The system used for this test was a $4 \mathrm{~K}$ cryostat and the entire process took approximately six minutes. This is quite reasonable, especially when compared to the time it takes to implement a Z-N test. The Z-N open loop test requires a large amount of process time because the user has to wait for equilibrium to be reached to accurately measure the gain. The closed loop version of the Z-N method requires that $\mathrm{P}$ be successively increased until self-oscillation occurs. This is also quite time consuming as it requires many cycles to determine a $\mathrm{P}$ value. The SI Method attempts to derive the essential information from these methods without ever requiring equilibrium during the test.

The SI Method proves to be quite easy to implement. With only three nonequilibrium step tests it is possible to collect the two peak overshoot values and the two times associated with the first peak and dip of oscillation. The measurement of these values is easy to program and is sufficient for the calculations defined above. The SI Method is far easier to implement than a Z-N test because of the difficulty in specifying and measuring a state of equilibrium. The closed loop Z-N P test also requires the induced 
oscillations to be sustained and non-decaying which can be difficult to measure systematically.
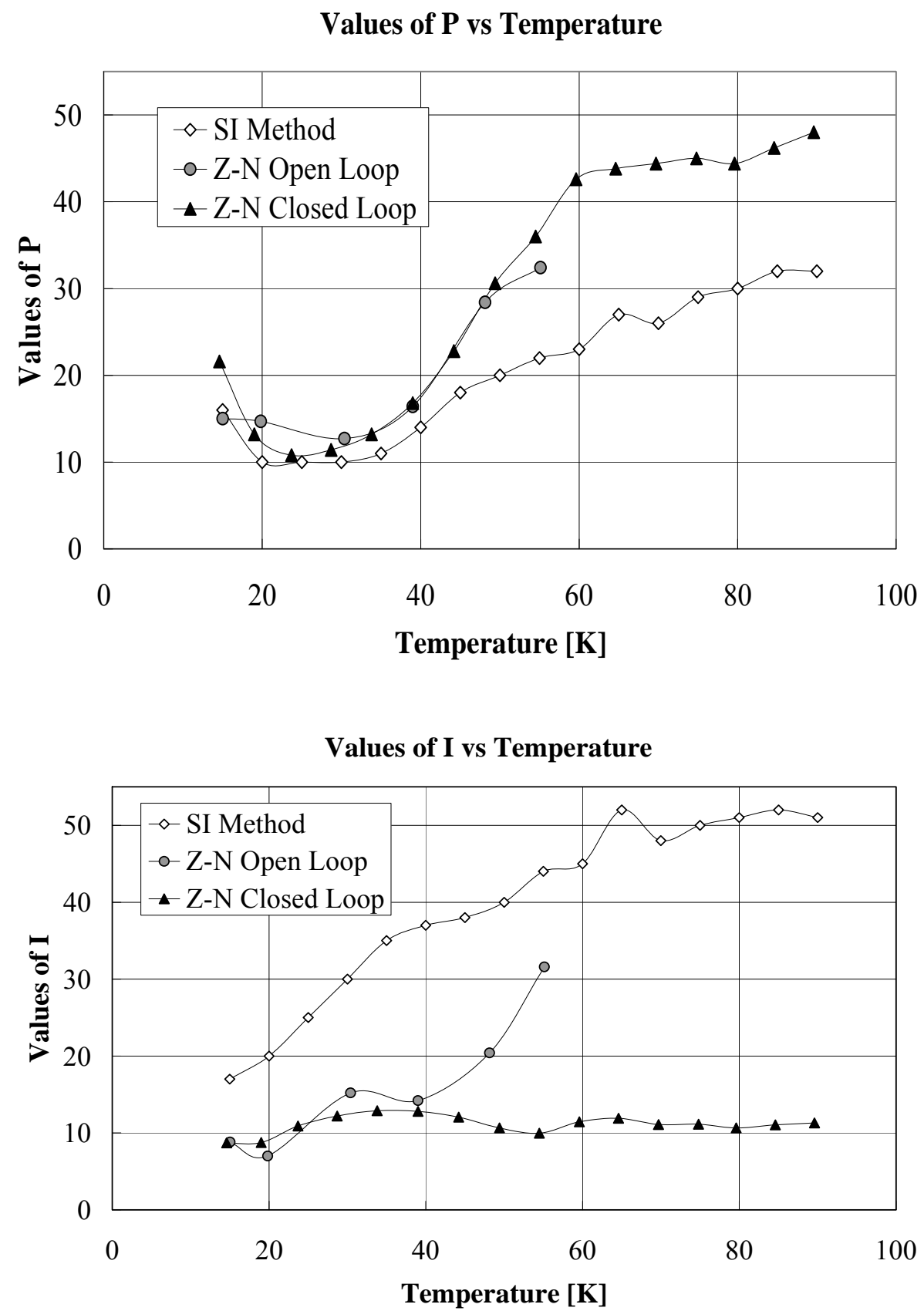

FIGURE 3 Values of $\mathrm{P}$ and I as a function of temperature. The values determined by the SI method are compared to those found by the method of Ziegler and Nichols implemented in both open and closed-loop variations.

The SI Method seems to perform well in comparison to other tuning methods even with the limited amount of data used to execute the algorithm. This result is primarily due to the fact that the Z-N methods were developed to provide accurate tuning of any process regardless of the nature of its process. The SI Method seems to do more with less because it is intended for use only with cryogenic systems. It may be of interest to others involved in process control to determine specifically what information is lost through the abbreviated test protocol. The easiest avenue to this end is through computer simulations. 


\section{CONTROL SIMULATION RESULTS}

Generally speaking, simulations allow access to a much larger parameter space than experimental studies. Simulations also allow exploration of hypothetical situations that may be difficult or impossible to create in the laboratory. For instance, certain nonlinear effects may be studied in a selective manner or all nonlinearities can be removed in an attempt to uncover the basic mechanisms of the control algorithm.

Early attempts at simulating the SI Method did not yield meaningful results [7]. This was primarily due to the use of a pure time delay in the model assumptions. A pure time delay is typically used to represent all of the fast time constants in a system. It was discovered that the short time constants had to be represented individually to accurately model the cryogenic system. The simulated linear approximation of the model was conveniently represented by one slow and two identical fast time constants. The most significant results of the simulation came from exploring the range of the ratio of slow to fast time constants, $\tau_{1} / \tau_{\mathrm{f}}$, the number of fast time constants, and various tweaking algorithms that could be employed to improve performance. The simulator employed the SI tuning method while simultaneously collecting a steady-state response so that any resulting instabilities could be observed.

The ratio of time constants for the system used in FIGURE 1 was found to be approximately ten at all temperatures [7]. Since this is a reasonable value for a typical cryogenic system, we will use this as the starting point of reference. For a fixed initial value of $\mathrm{P}$, the algorithm produced reasonable values for $\mathrm{P}, \mathrm{I}$, and $\mathrm{D}$ for ratios up to 33 . As the ratio increased the peak overshoot was reduced. This continued until the control test no longer resulted in an overshoot and the test failed. If the initial $\mathrm{P}$ value was increased, the maximum ratio would also increase. It was found that there always exists a "large enough" $\mathrm{P}$ value such that an overshoot is guaranteed. There exists no criterion in the SI Method to determine if the initial P value is "large enough" and it is left to the user to adjust this before attempting a second tuning experiment. The same difficulty can arise as the ratio of time constants is lowered towards one. When the ratio reached a value of three, some of the $\mathrm{P}$ control tuning tests resulted in instability. This could be avoided by reducing the initial $\mathrm{P}$ value used for test. With a suitable choice of initial values, it was even possible to tune a simulated system with a ratio of less than one. This would represent a system with two slow and one fast time constant, a situation that is hard to envision in a cryogenic environment. This seems to suggest that the SI Method is applicable to systems over a wide range of scales and that the initial value of $\mathrm{P}$ is of significance to the quality of the resultant tuning.

The number of fast time constants used in the above simulation was $\mathrm{N}=2$. It is of interest to find out if the results could be valid for other values. Values of $\mathrm{N}=3$ and $\mathrm{N}=4$ were examined with the simulator. It was found that the quality of the resulting parameters depended heavily on the values of P, I, and D. Stable results could be found only after adjusting the I and D values up and the P value down. The SI Method only produced reliable results for $\mathrm{N}=2$.

The simulator allowed for various tweaking strategies to be tested after the tuning process was completed. A complete list of these strategies and their results are listed elsewhere [7]. It was found that such strategies can be of value to users striving for improved performance or for a specific performance characteristic such as minimum overshoot or fastest time to equilibrium. 


\section{CONCLUSIONS}

The SI Method has proven to provide acceptable tuning parameters for a wide variety of cryogenic apparatus. The algorithm is robust against procedural inaccuracies,

simple to apply, and quick to produce results. The cost of such efficiency appears to be the limitation of the methods applicability to systems with one slow and exactly two fast time constants. The tuning technique also seems to be somewhat sensitive to the initial choice of the $\mathrm{P}$ value. These shortcomings may be addressed by implementation of an "Improved SI Method" that samples additional features of the step response at the cost of somewhat longer processing time. An advanced algorithm could also include an automated tweaking option to guide the user closer to "optimum" performance.

\section{ACKNOWLEDGEMENTS}

The digital control code for the M9700 was developed by Mark Sheats (Scientific Instruments). Open loop tests and comparative experimental studies against the ZieglerNichols method were performed by Elizabeth Strehlow (Purdue University). Development of the Matlab ${ }^{T M}$ code for the simulator was done by Jon Burford (Florida Atlantic University).

\section{REFERENCES}

1. Forgan, E.M., Cryogenics, 14, pp. 207-214 (1974).

2. Ziegler, J.G., Nichols, N.B., "Optimum Settings for Automatic Controllers", Transactions of the ASME, 64, 1943, pp 759-768.

3. Ogata, K., Modern Control Engineering, Third Edition, Prentice Hall, New Jersey, 1997, pp. 669-674.

4. M. Zhuang and D.P. Atherton, "Automatic tuning of optimum PID controllers", IEE Proceedings-D, 140, 1993, pp.216-224.

5. Voda, A., Landau, I.D., Automatica, 31, 1995, pp. 41-53.

6. Sheats, D.C., Roth, Z.S., Sheats, M., Strehlow, E., “Autotune of PID Cryogenic Temperature Control Based on Closed Loop Step Response Tests", Proceedings of the $17^{\text {th }}$ Conference on Recent Advances in Robotics, edited by Alexander Leonessa, University of Central Florida, Orlando, FL, 2004, CD proceedings.

7. Sheats, D.C., Roth, Z.S., Burford, J.K., Snyder, J.W., "Simulation Studies of the SI PID Control Autotuning", Proceedings of the $18^{\text {th }}$ Conference on Recent Advances in Robotics, edited by Carl Crane, University of Florida, Gainesville, FL, 2005, CD Proceedings. 